\title{
PROGNOSTIC ACCURACY OF SOFA SCORE AND QSOFA AS A PREDICTOR OF MORTALITY AMONG SEPSIS PATIENTS PRESENTING TO EMERGENCY DEPARTMENT IN ONE OF A TERTIARY HOSPITAL IN KATHMANDU, NEPAL
}

\author{
Thapa $S,{ }^{1}$ Upreti $A R,{ }^{2}$ Bajracharya $R,{ }^{1}$ Lingden $B K^{1}$
}

${ }^{1}$ Department of General Practice and Emergency Medicine, ${ }^{2}$ Clinical Pharmacist, Nepal Medical College \& Teaching Hospital, Attakhel, Gokarneshwor-8, Kathmandu, Nepal

\begin{abstract}
There are many clinical scoring systems that measure the severity in sepsis and septic shock. Therefore, our study aims to calculate prognostic accuracy of commonly used scoring system SOFA and qSOFA in emergency department as a predictor of mortality among sepsis patients.This was prospective observational study conducted in an emergency department for a period of seven months. 156 patients were studied and descriptive statistical analysis was done. The most common source of infection was respiratory. A positive and moderate correlation was seen between initial SOFA score and qSOFA score. The AUC of SOFA score and qSOFA for predicting the mortality were 0.978 and 0.886 with sensitivity of $96.9 \%$ and specificty of $57 \%$ for SOFA and sensitivity of $96.9 \%$ and specificity of $76.1 \%$ for qSOFA.SOFA and qSOFA both proved to be similar as a simple prognostic tool with discriminatory capacity in predicting prognosis in septic patient presenting to emergency department.
\end{abstract}

\section{KEYWORDS}

Mortality, sepsis, QSOFA, SOFA

\section{CORRESPONDING AUTHOR}

Dr. Sameer Thapa

Department of General Practice and Emergency Medicine,

Nepal Medical College Teaching Hospital,

Attarkhel, Gokarneshower-8, Kathmandu, Nepal

Email: sams.thp@gmail.com

Orcid ID: 0000-0002-0922-2289

DOI: https://doi.org/10.3126/nmcj.v22i1-2.30025 


\section{INTRODUCTION}

Sepsis-3 defines sepsis as life-threatening organ dysfunction caused by a dysregulated host response to infection. ${ }^{1}$ It has been one of the major causes for morbidity and mortality worldwide with an increase in reported incidence. ${ }^{2}$ In order to decrease mortality in emergency department it is important to develop accurate and reliable methods to assess severity of sepsis favoring prompt initiation of appropriate therapy. ${ }^{3,4}$

Presently, there are many clinical scoring systems that measure the disease severity in septic population. Many of these scores such as Acute Physiology and Chronic Health Evaluation Score, Simplified Acute Physiology Score III, Logistic Organ Dysfunction Score, the Mortality Probability Model III, were actually derived and validated in the intensive care unit (ICU). ${ }^{5,6}$ They are time consuming and required information that is not readily available in emergency setting. Previous investigations have also demonstrated these scores to be inadequate when applied to emergency department patients. ${ }^{7}$

Together with the publication of the Third International Consensus Definitions for Sepsis and Septic Shock (Sepsis-3), a simplified Sequential Organ Failure Assessment (SOFA) score coined quick SOFA (qSOFA), which was proposed to help clinicians identify sepsis patients among those with suspected infection. ${ }^{8}$ In a recent prospective study conducted in emergency departments of four European countries, qSOFA had greater prognostic accuracy for in-hospital mortality than SIRS or severe sepsis, but not SOFA. ${ }^{9}$ Further implementation of this within existing guidelines for sepsis is yet to be seen. ${ }^{10}$ Therefore, our study aims to calculate prognosis accuracy of SOFA and qSOFA in Emergency Department as a predictor of mortality among sepsis patients.

\section{MATERIALS AND METHODS}

This was prospective observational study conducted in emergency department of Nepal Medical College and Teaching Hospital from January 2019 to August 2019 on 156 patient age more than 16. Ethical clearance was taken from the Institutional Review Committee. All patients presenting to the emergency department with suspected infection with qSOFA score of 2 or more points were enrolled in the study.

The qSOFA score was calculated as soon as patient with suspected infection enter in the emergency rooms. Later, after getting Lab result of the patient SOFA score was calculated. Written consent was obtained from participants.
Patients with an age less than 16 years, do-notresuscitate order, acute coronary syndrome, leave against the medical advice, incomplete information and data and who deny consent were excluded. The patients were followed up by phone calls at 28 days for mortality status. Collected data were entered in Microsoft excel and analyzed in SPSS version 16. Level of Significance for all analytical tests was set 0.05 and $p$ value $<0.05$ was considered significant. Spearman's correlation analysis was done to correlate SOFA and qSOFA. ROC curve was drawn for SOFA and qSOFA to calculate AUC, sensitivity and specificity.

\section{RESULTS}

The sample size in our study was 156 patients. 73 were male and 83 were female. The age distribution was between 16 to 85 years with mean age being $51.26 \pm 23.3$. The most common source of infection was respiratory $(65 \%)$ as shown in Table 1.

Table 1: Distribution of Source of Infection

\begin{tabular}{|lcc|}
\hline Source & Frequency & $\mathbf{\%}$ \\
\hline Respiratory & 65 & 41.7 \\
CNS & 26 & 16.6 \\
Gastrointestinal & 23 & 14.7 \\
Genitourinary & 12 & 7.7 \\
Skin \& Musculoskeletal & 2 & 1.3 \\
Others & 28 & 17.9 \\
\hline Total & $\mathbf{1 5 6}$ & $\mathbf{1 0 0 . 0}$ \\
\hline
\end{tabular}

Among 156 patients, 64 patients had mortality when followed up for 28 days, mortality percentage being $41.5 \%$.

\begin{tabular}{|c|c|c|c|}
\hline Outcome & $\begin{array}{l}\text { Mean } \pm \text { SD } \\
\text { SOFA score }\end{array}$ & $\mathrm{N}$ & $\begin{array}{c}\text { P-value } \\
\text { (chi-sq. test) }\end{array}$ \\
\hline Non Survivors & $12.70 \pm 2.81$ & 64 & 0.00 \\
\hline Survivors & $4.52 \pm 2.33$ & 90 & \\
\hline Total & $7.88 \pm 4.76$ & 154 & \\
\hline
\end{tabular}

SOFA score at presentation were compared between survivors and non survivors as shown in Table-2 and the score was significantly higher in non survivors $(\mathrm{P}<0.05)$. Univariate analysis revealed that females were more likely to be non survivors $(\mathrm{p}<0.05)$.

A positive and moderate correlation was seen between initial SOFA score and QSOFA score. Spearman's rho correlation coefficient $(r 2)$ was $0.544(\mathrm{p}<0.05)$. 
The AUC of SOFA score for predicting the mortality was 0.978 . The best cut off value was with 8.50 with sensitivity of $96.9 \%$ and specificty of $57 \%$. (Fig. 1)

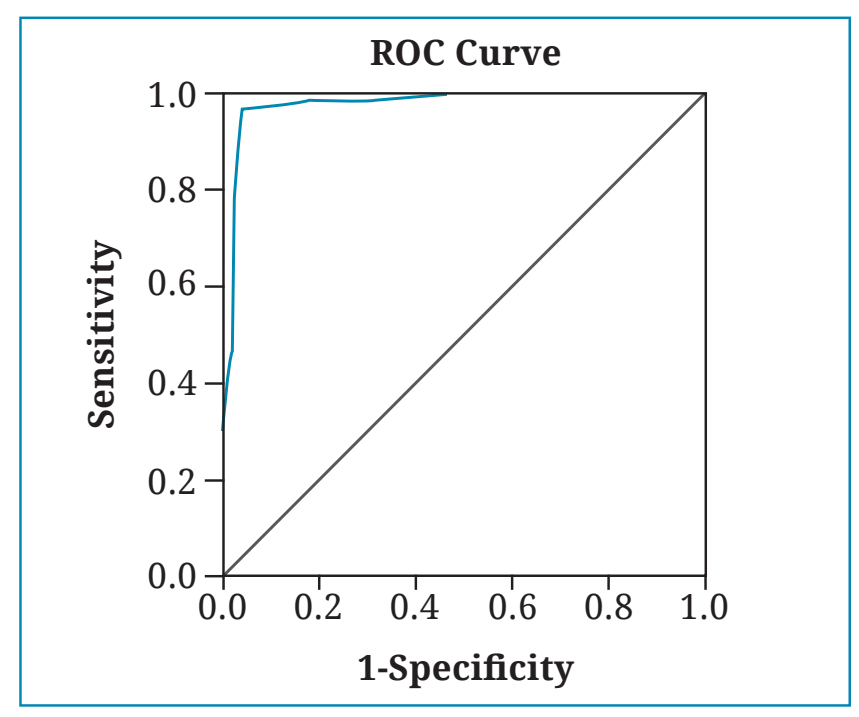

Fig. 1: ROC curve of SOFA

The AUC of qSOFA score for predicting the mortality was 0.886 . The best cut off value was with 1.50 with sensitivity of $96.9 \%$ and specificity of $76.1 \%$. (Fig: 2 )

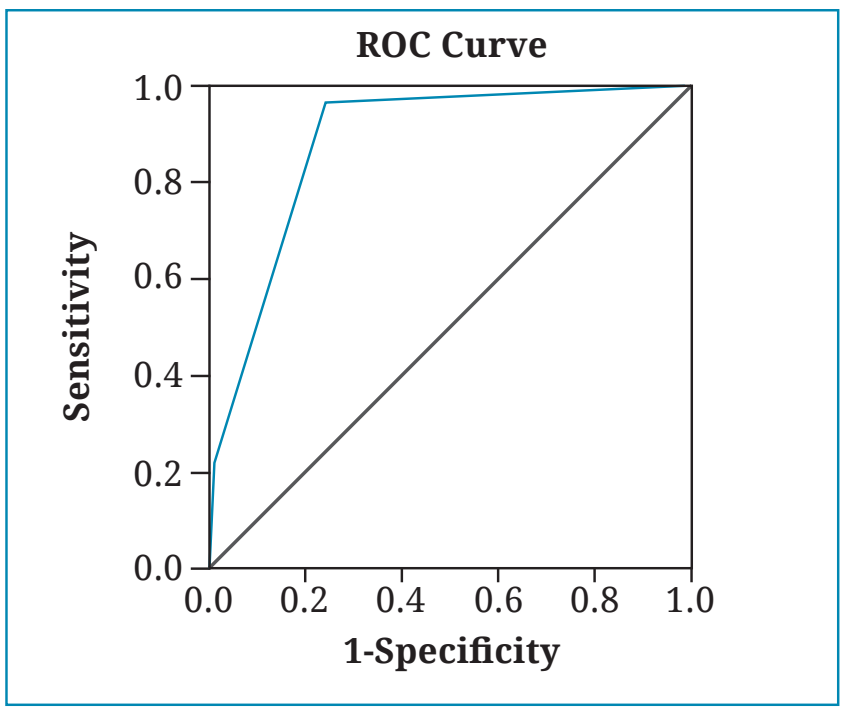

Fig. 2: ROC curve of QSOFA

\section{DISCUSSION}

The Sequential Organ Failure Assessment (SOFA) is used to assess the severity of dysfunction in six organ systems (Pulmonary, coagulation, hepatobiliary, cardiovascular, renal, and neurologic). ${ }^{11}$ whereas The quick Sequential Organ Failure Assessment (qSOFA) is used to enables rapid risk stratification of septic patients.
In our study, there was predominance of female population, respiratory system being the most common source of infection in the study population. This result is consistent with the similar study done in Nepal. ${ }^{12,13}$ The mortality was $41.5 \%$ in our study whereas in the study done in same setting in Nepal, the mortality rate from sepsis was $36.5 \%{ }^{13}$ and $22.92 \%^{12}$ respectively. The initial SOFA score were significantly higher in non survivors when compared to survivors. Similar results were seen in studies by Nair et $a l^{14}$ Shrestha et al. ${ }^{15}$

In our study a positive and moderate correlation was seen between initial SOFA score and qSOFA score. The study done by Garbero et al ${ }^{16}$ concluded that qSOFA is a weak tool for predicting for prognosis in the emergency department.

Our study calculated higher sensitvity (96.9\%) and specificty ( $57 \%$ ) of SOFA at the cut of value of 8.50 which slightly differ from the study done by Rajbhandari et al where the sensitivity and specificity was calculated to be $84.8 \%$ and $73 \%$ respectively at the cut of value of $6.50 . .^{12}$

In a study done by Garbero et $a l^{16}$ in emergency department, sensitivity of SOFA was $93.7 \%$ for mortality. In our study The AUROC of SOFA is 0.978 . In a study done by Chen et $a l^{17}$ the value of AUROC for initial SOFA was 0.917 which is almost similar to our study.

In contrast, the AUROC for SOFA was found to be 0.63 in one of the study done in the emergency department of a low middle income country. ${ }^{18}$ The AUROC of qSOFA score for predicting the mortality was 0.886 with $96.9 \%$ sensitivity and $76.1 \%$ specificity at the cut off value of 1.50 which is somewhat similar to the study done by Baig MA et $a l^{18}$ where the AUROC of qSOFA for predicting mortality in subjects was 0.89 with $92 \%$ sensitivity and $85 \%$ specificity. In contrast Hyadar et $a l^{19}$ concluded that qSOFA has a sensitivity of $39 \%$ within 28 days.

Our study has several limitations, this study was conducted in a single center so the results may not be generalized to other centers. Larger multicentered studies and evaluation of special category of patients may be helpful. Our analysis focused only on initial score of SOFA and qSOFA on admission and not the subsequent scores.

Both SOFA and qSOFA can be used as a tool in predicting mortality among sepsis patients presenting to emergency department in resources limited countries like ours. Even though both tools are simple and readily available, further studies with greater number of patients are required to confirm their definite value as a prognostic tool in sepsis patients visiting emergency department. 


\section{REFERENCES}

1. Singer M, Deutschman C, Seymour C, et al. The third international consensus definitions for sepsis and septic shock (sepsis-3). J Am Med Assoc 2016; 315: 801-10.

2. Chun-Ta Huang ,Sheng-Yuan Ruan ,Yi-Ju Tsai ,Shih-Chi Ku , Chong-Jen Yu .Clinical Trajectories and Causes of Death in Septic Patients with a Low APACHE II Score.Settings. J Clin Med 2019; 8: 1064.

3. Seymour CW, Liu VX, Iwashyna TJ, Brunkhorst FM, Rea TD, Scherag A, et al. Assessment of Clinical Criteria for Sepsis: For the Third International Consensus Definitions for Sepsis and Septic Shock (Sepsis-3). J Am Med Assoc 2016; 315: 762-74.

4. Rhodes A, Evans LE, Alhazzani W, Levy MM, Antonelli M, Ferrer R, et al. Surviving Sepsis Campaign: International Guidelines for Management of Sepsis and Septic Shock: 2016. Intens care Med 2017; 43: 304-77.

5. Metnitz PGH, Moreno RP, Almeida E, et al. SAPS 3 from evaluation of the patient to evaluation of the intensive care unit. Part 1: objectives, methods, and cohortdescription.Intens CareMed2005;31:1336-44.

6. Higgins TL, Teres D, Copes WS, et al. Assessing contemporary intensive care unit outcome: an updated Mortality Probability Admission Model (MPM0-III). Crit Care Med 2007; 35: 827-835

7. Jones AE, Fitch MT, Kline JA. Operational performance of validated physiologic scoring systems for predicting in-hospital mortality among critically ill emergency department patients. Crit Care Med 2005; 33: 974-78.

8. Seymour CW, Liu VX, Iwashyna TJ, Brunkhorst FM, Rea TD, Scherag A, et al. Assessment of Clinical Criteria for Sepsis: For the Third International Consensus Definitions for Sepsis and Septic Shock (Sepsis-3). J Am Med Assoc 2016; 315: 762-74.

9. Freund Y, Lemachatti N, Krastinova E, Van Laer $\mathrm{M}$, Claessens YE, Avondo A, et al. Prognostic Accuracy of Sepsis-3 Criteria for In-Hospital Mortality Among Patients With Suspected Infection Presenting to the Emergency Department. J Am Med Assoc 2017; 317: 301-8.

10. Antonelli $\mathrm{M}$, DeBacker $\mathrm{D}$, Dorman $\mathrm{T}$, et al. Surviving Sepsis Campaign Responds to Sepsis-3. Surviving sepsis Campaign; 2016. Website. Available at: http://www. survivingsepsis.org/ SiteCollectionDocuments/SSC-Statements-SepsisDefinitions-3- 2016.pdf (Access on: 28 $8^{\text {th }}$ Sep, 2018)
11. Vincent JL, Moreno R, Takala J et al. The SOFA (Sepsis-related organ failure assessment) score to describe organ dysfunction/failure. On behalf of the working group on sepsis-related problems of the european society of intensive care medicine. Intens Care Med 1996; 22: 707-10.

12. Rajbhandari P, Shakya YL, Upreti AR. Neutrophil to Lymphocytic count and SOFA score as prognostic marker in patient with sepsis. Nepal Med J 2019; 01: 43-7.

13. Lamichhane, S, Manandhar, N, Dhakal, S, Shakya YL . Management and Outcome of Severe Sepsis and Septic Shock Patients. JNHRC 2018; 16: 165-71.

14. Nair R, Bhandary NM, D’Souza AD. Initial Sequential Organ Failure Assessment score versus Simplified Acute Physiology score to analyze multiple organ dysfunction in infectious diseases in Intensive Care Unit. Indian J Crit Care Med 2016; 20: 210-15.

15. GS Shrestha, $\mathrm{R}$ Gurung and $\mathrm{R}$ Amatya. Comparison of acute physiology, age, chronic health evaluation III score with initial sequential organ failure assessment score to predict ICU mortality. Nepal Med Coll J 2011; 13: 50-4.

16. Garbero RF, Simões AA, Martins GA, Cruz LVD, von Zuben VGM. SOFA and qSOFA at admission to the emergency department: Diagnostic sensitivity and relation with prognosis in patients with suspected infection. Turk J Emerg Med 2019; 19: 106-10.

17. Chen YC, Tian YC, Liu NJ et al. Prospective cohort study comparing sequential organ failure assessment and acute physiology, age, chronic health evaluation III scoring systems for hospital mortality prediction in critically ill cirrhotic patients. Int'l J Clin Pract 2006; 60: 160-6.

18. Baig MA, Sheikh S, Hussain E, Bakhtawar S, Subhan Khan M, Mujtaba S, Waheed S. Comparison of qSOFA and SOFA score for predicting mortality in severe sepsis and septic shock patients in the emergency department of a low middle income country. Turk J Emerg Med 2018; 18: 148-51.

19. Haydar S, Spanier M, Weems P, Wood S, Strout T. Comparison of QSOFA score and SIRS criteria as screening mechanisms for emergency department sepsis. Am J Emerg Med 2017; 35: 1730-33. 